\title{
PROPOSTA PARA MELHORIA DA EFICIÊNCIA HIDROENERGÉTICA DE DESTILADOR
}

\author{
Renato Campos ${ }^{1}$; Eduardo Cohim ${ }^{1}$; Samuel Sipert' ${ }^{1}$; Hamilton de Araújo Silva ${ }^{1}$
}

1 Universidade Estadual de Feira de Santana; Feira de Santana/Bahia; renatocampos.ec@gmail.com

Resumo: Neste trabalho faram apontadas soluções para melhoramento da eficiência em destiladores de pequeno porte, obtendo através da avaliação do ciclo de vida (ACV), o consumo energético do destilador convencional e, comparando-o com o do cenário proposto, acrescido do custo energético das mudanças propostas diluídas ao longo da vida útil do equipamento. No sistema proposto foi sugerido um resfriador geotérmico possibilitando a reutilização da água de resfriamento do condensador e um aquecedor solar com baixo custo de implantação, elevando a temperatura de entrada no destilador. O sistema proposto apresentou uma redução no consumo energético de $34,2 \%$ por litro de água destilada produzida, o consumo de água associada a destilação foi reduzido de $8 \mathrm{~L}$ para zero.

Palavras-Chave: Destiladores; Avaliação do ciclo de vida; Eficiência hidro energética.

\section{PROPOSAL FOR IMPROVEMENT OF DISTILLER HYDROENERGY EFFICIENCY}

\begin{abstract}
In this work, solutions for improving the efficiency of small distillers were pointed out, obtaining through the Life Cycle Assessment (LCA), the energy consumption of the conventional distiller and, comparing it with the proposed scenario, plus the energy cost of the changes. proposals diluted over the life of the equipment. In the proposed system, a geothermal chiller was suggested, enabling the reuse of condenser cooling water and a low cost solar heater, increasing the inlet temperature in the distiller. The proposed system presented a reduction in energy consumption of $34.2 \%$ per liter of distilled water produced, the water consumption associated with distillation was reduced from $8 \mathrm{~L}$ to zero.
\end{abstract}

Keywords: Distillers; Life cycle assessment; Hydro energy efficiency. 


\section{INTRODUÇÃO}

Devido ao atual cenário de escassez de recursos, se torna cada vez mais importante a melhoria da eficiência energética dos sistemas geradores de impacto ambiental. A avaliação do ciclo de vida proporciona uma visão holística dos aspectos ambientais e energéticos associados a um serviço ou produto, demonstrando através de medidas que expressam os resultados referentes ao desempenho energético e apoiando as estratégias ambientalmente melhores [1].

A ACV vem se constituindo, cada vez mais, uma importante ferramenta da Gestão Ambiental, embora esteja ainda em fase de consolidação no que se refere a alguns de seus componentes. O interesse despertado pela ACV tem aumentado pelo fato de permitir através de um foco de abordagem sistêmico, tanto a identificação de oportunidades de melhoria do desempenho ambiental de um produto ou processo quanto a comparação ambiental de mais de um deles, desde que desempenhem a mesma função [2].

Conforme [3, 4] a avaliação do ciclo de vida considera todo o ciclo de vida de um produto, desde a extração e aquisição de matérias-primas, através da produção de energia e materiais, manufatura, uso, tratamento de resíduos no fim de vida até a disposição final. Os estudos de ACV incluem quatro fases: definição de objetivo e escopo, análise de inventário, avaliação de impacto e interpretação de resultados.

Os destiladores de pequeno porte comumente utilizados em laboratórios de pesquisa e ensino, possuem um baixo custo de aquisição e manutenção. Em contrapartida esses modelos apresentam um grande desperdício, já que a água utilizada para resfriamento do condensador é descartada. Outro aspecto que deve ser levado em conta é o alto consumo energético destes equipamentos devido a utilização de uma resistência para aquecimento do fluido no reator [5].

A preocupação com os destiladores deve ser motivada pelo elevado número de equipamentos existentes no campus universitário, onde verificou uma perda de água durante o processo de destilação de cerca de 50 L/L de água destilada [6].

Neste contexto este artigo tem o objetivo de analisar e propor soluções para melhorar a eficiência de destiladores de pequeno porte, que apesar de apresentar um grande desperdício de água, são amplamente utilizados por seu baixo custo de aquisição e implantação. Através da avaliação do ciclo de vida foram analisados e comparados dois cenários, sendo o primeiro um sistema convencional e o segundo um sistema proposto visando a redução de consumo energético, utilizando tecnologias de baixo custo.

\section{METODOLOGIA}

A primeira etapa do estudo foi a determinação do modelo de destilador a ser analisado, sendo escolhido o modelo MA078/10 da MARCONI, mesmo aparelho utilizado no laboratório de tecnologia da UEFS. A partir dos dados de consumo (água utilizada para o resfriamento do condensador) e produção fornecidos pelo fabricante 
foi possível determinar o cenário convencional. Para esta análise foi utilizado o SimaPro e adotada uma produção semanal de $200 \mathrm{~L}$ de água destilada e uma vida útil do equipamento de 15 anos.

Segundo site do fabricante o destilador em questão apresenta uma produção de 10L/h de água destilada, sendo que a vazão consumida para resfriamento da serpentina é de $80 \mathrm{~L} / \mathrm{h}$. Para este trabalho a unidade funcional adotada foi um litro de água destilada, ou seja, para cada litro de água destilada são utilizados $8 \mathrm{~L}$ de água para o resfriamento da serpentina e, em seguida, são descartados.

O primeiro cenário analisado foi o convencional, onde a água a ser destilada entra no reator com temperatura ambiente $\left(25^{\circ} \mathrm{C}\right)$ e é elevada a $100^{\circ} \mathrm{C}$. Para determinar a potência elétrica consumida pelo destilador calcula-se na Equação $1 \mathrm{a}$ energia dissipada (E) necessária para aquecer 1 litro de água, de $25^{\circ} \mathrm{C}$ para $100^{\circ} \mathrm{C}$.

$$
\begin{array}{r}
Q=m * c * \Delta T \\
Q=E
\end{array}
$$

$\mathrm{Q}=$ quantidade de calor (kcal); $\mathrm{m}=$ massa de água $(\mathrm{kg}) ; \mathrm{c}=$ calor específico $\left(\mathrm{kcal} /{ }^{\circ} \mathrm{C}\right) ;=$ variação de temperatura; $\mathrm{E}=$ energia dissipada $(\mathrm{kWh})$.

Além da energia elétrica consumida, foi somada ao consumo energético do cenário convencional a energia incorporada ao tratamento e distribuição da água de resfriamento da serpentina, já que a mesma é descartada. Segundo Guanais et al. (2016), a demanda energética acumulada do Sistema Integrado de Abastecimento de Água de Feira de Santana foi de $3,51 \mathrm{kWh} / \mathrm{m}^{3}$ de água consumida, considerando a energia primaria.

Visando a melhoria da eficiência do destilador, foram sugeridas duas melhorias no cenário proposto, na primeira, mostrada na Figura 1, um sistema de aquecimento solar de água eleva a temperatura de entrada na caldeira do destilador, de $25^{\circ} \mathrm{C}$ para $50^{\circ} \mathrm{C}$, diminuindo a quantidade de energia elétrica consumida para que a água entre em ebulição, o aquecedor solar foi dimensionado a partir da NBR 15569 [7].

Figura 1. Esquema isométrico para o aquecedor solar

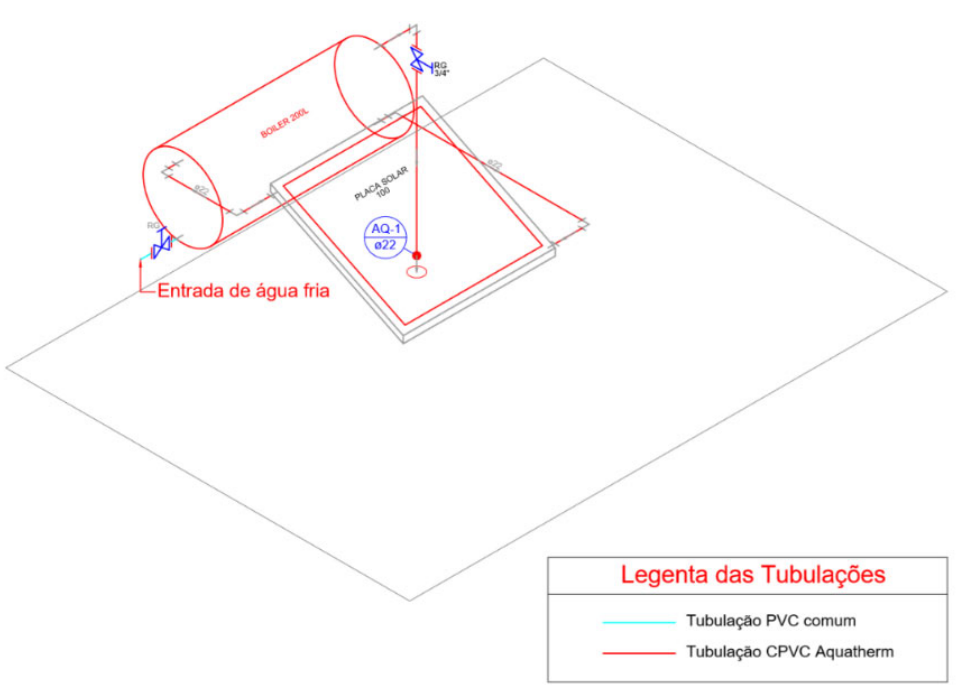


A segunda melhoria elimina a necessidade de descarte da água de resfriamento, seguindo o modelo utilizando por [8], foi dimensionado um resfriador geotérmico, diminuindo a temperatura da água, de $75^{\circ} \mathrm{C}$ na entrada da serpentina para $25^{\circ} \mathrm{C}$ na sua saída como mostra a Figura 2, possibilitando a sua recirculação. A profundidade da serpentina foi de $3 \mathrm{~m}$ da superfície do solo, composta por $14 \mathrm{~m}$ de tubo de cobre flexível com 1/2" (Classe 1). Em série com o resfriador geotérmico foi colocado um reservatório de acumulação para a água de resfriamento e uma bomba para recalque da água até o ponto de utilização.

A partir do dimensionamento dos sistemas de aquecimento solar e resfriamento geotérmico do cenário proposto foi possível elaborar o projeto mostrado na Figura 1 e determinar os quantitativos de materiais, necessários para a construção do inventario (Tabela 1).

Tabela 1. Inventário de materiais para cenário proposto

\begin{tabular}{lccc}
\multicolumn{1}{c}{ Lista de Materiais } & Quantidade & $\begin{array}{c}\text { Massa por } \\
\text { unidade }\end{array}$ & $\begin{array}{c}\text { Total de } \\
\text { massa ou } \\
\text { unidades }\end{array}$ \\
\hline \multicolumn{1}{c}{ AQUECEDOR SOLAR DE ÁGUA } & & $2,44 \mathrm{~kg}$ \\
\hline CPVC Aquatherm 22mm & $18,09 \mathrm{~m}$ & $0,135 \mathrm{~kg} / \mathrm{m}$ & $1 \mathrm{Unid}$. \\
\hline $\begin{array}{l}\text { Placa Solar - CSC Premium - Cumulus 100 } \\
\text { Reservatório térmico solar - Horizontal 200L }\end{array}$ & $1 \mathrm{pç}$ & Unid. & $1 \mathrm{Unid}$. \\
\hline \multicolumn{1}{|c|}{ RESFRIADOR GEOTÉRMICO } & Unid. & \\
\hline Reservatório cilíndrico Tigre - Polietileno 310L & $1 \mathrm{pç}$ & $7,50 \mathrm{~kg} /$ Unid. & $7,50 \mathrm{~kg}$ \\
\hline PVC rígido soldável 20mm & $13.54 \mathrm{~m}$ & $0,135 \mathrm{~kg} / \mathrm{m}$ & $1,83 \mathrm{~kg}$ \\
\hline Cobre - Tubos de cobre 15mm & $14 \mathrm{~m}$ & $0,280 \mathrm{~kg} / \mathrm{m}$ & $3,92 \mathrm{~kg}$ \\
\hline Bomba Hidráulica Dancor - CAM-2 1/10CV & $1 \mathrm{pç}$ & Unid. & $1 \mathrm{Unid}$. \\
\hline
\end{tabular}


Figura 2. Esquema isométrico para o resfriador geotérmico

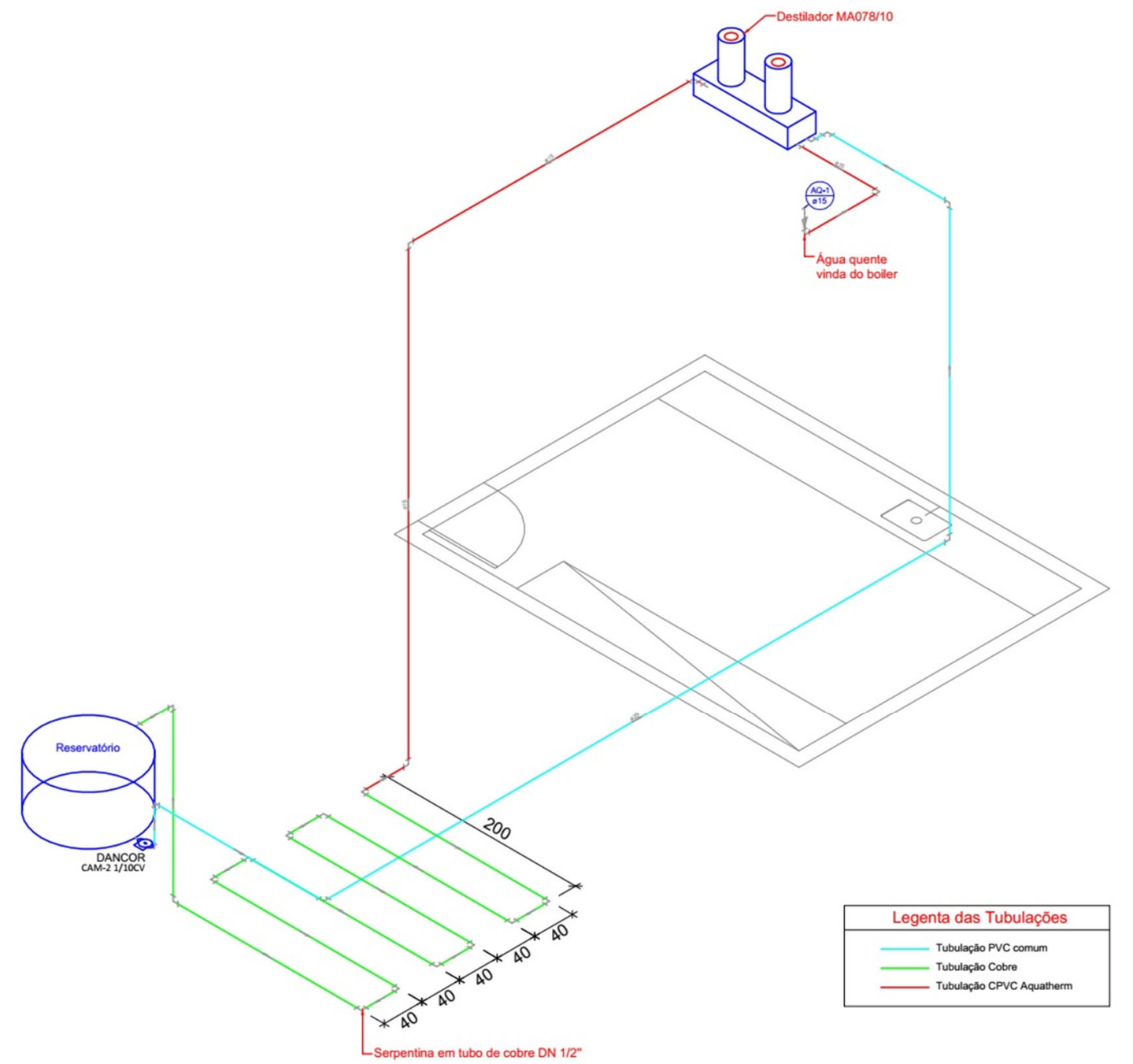

Como apresentado na Tabela 1 toda a instalação de água quente é composta de CPVC Aquatherm, representada em vermelho na Figura 1, a placa solar e o boiler foram locados sobre a cobertura, para água fria foi usado o PVC Marrom, representado em azul, em verde está representada a serpentina composta de tubo de cobre.

A determinação da potência elétrica consumida pelo destilador no cenário proposto foi calculada a partir da equação 1 adotando rendimento $=90 \%$, assim como no cenário convencional, porém levando em conta uma variação de temperatura de $50^{\circ} \mathrm{C}$ ao invés de $75^{\circ} \mathrm{C}$, este consumo foi somado ao gerado pela bomba hidráulica, com um rendimento de $25 \%$, de acordo com equação 2 demonstrada por Guanais (2016):

$$
E=0,00273 * \frac{H * V}{\eta}
$$

$\mathrm{E}=$ energia dissipada (kWh); $\mathrm{H}=$ altura manométrica (m.c.a); $\mathrm{V}=$ volume de água bombeada $\left(\mathrm{m}^{3}\right) ; \eta=$ rendimento total do conjunto motor-bomba. 


\section{RESULTADOS E DISCUSSÃO}

Considerando que a quantidade de água consumida pelo destilador é $8 \mathrm{~L} / \mathrm{L}$ no cenário convencional foi possível calcular a energia associada à sua produção (cadeia produtiva de abastecimento) e soma-la com o consumo de energia elétrica do destilador, sendo que no software SimaPro a energia secundária consumida no destilador foi convertida em primaria antes de ser somada à energia associada ao abastecimento de água de Feira de Santana-BA apontada por Guanais et al. (2016).

O cenário proposto, que leva em conta as duas modificações em conjunto, obteve um valor reduzido de consumo elétrico em relação ao convencional já que a água a ser destilada entra no reator a $50^{\circ} \mathrm{C}$, reduzindo a energia consumida para aquecimento. Além disso, foi observado que a energia agregada à produção e destinação final dos materiais utilizados para os sistemas de aquecimento solar e resfriador geotérmico não representou grande consumo energético, levando em conta a vida útil do aparelho. O consumo elétrico calculado para a bomba de recalque representou uma contribuição de impacto pouco representativa para o para o cenário proposto.

Tabela 2. Demanda de energia acumulada por litro de água destilada

\begin{tabular}{lccrr} 
& Quantidade & Unidade & Energia Primária (Wh/L) \\
\hline \multicolumn{1}{c}{ Cenário convencional } & & & \\
\hline Energia elétrica & 97 & Wh/L & 206,0 \\
\hline Consumo de água & 8 & $\mathrm{~L}$ & 28,1 \\
\hline Total & - & Wh/L & 234,1 \\
\hline \multicolumn{1}{c}{ Cenário modificado } & & & 138,3 \\
\hline Energia elétrica & 65 & Wh/L & 22,7 \\
\hline Energia fase de construção & - & - & 161,0 \\
\hline Total & - & Wh/L & \\
\hline
\end{tabular}

Comparando os dois cenários na Tabela 2 foi observado um consumo de 234,1 Wh/L para o cenário convencional e $161 \mathrm{Wh} / \mathrm{L}$ para o cenário proposto, representando uma economia energética de $31,2 \%$.

Tabela 3. Impacto energético da implantação dos sistemas.

\begin{tabular}{lrrr}
\hline & $\begin{array}{c}\text { Aquecimento } \\
\text { solar da água }\end{array}$ & $\begin{array}{c}\text { Resfriador } \\
\text { geotérmico }\end{array}$ & \multicolumn{1}{c}{ Total } \\
\hline Custo energético dos materiais $(\mathrm{Wh} / \mathrm{L})$ & 20,6 & 2,1 & 22,7 \\
\hline Energética subtraída do sistema $(\mathrm{Wh} / \mathrm{L})$ & 67,7 & 28,1 & 95,8 \\
\hline Economia energética total $(\mathrm{Wh} / \mathrm{L})$ & 47,1 & 26,0 & 73,1 \\
\hline
\end{tabular}


Analisando a Tabela 3 observou-se que o resfriador geotérmico apresenta um custo energético dos materiais muito baixo gerando uma economia total de $26 \mathrm{Wh} / \mathrm{l}$. Porém, apesar de apresentar um custo aproximadamente dez vezes maior que o resfriador geotérmico, o aquecimento solar se mostrou mais vantajoso em termos absolutos, já que subtrai mais energia elétrica demandada pelo processo de destilação, reduzindo o consumo energético em 47,1 Wh/L.

\section{CONCLUSÃO}

O sistema proposto para aumento da eficiência do processo de destilação de água apresentou uma redução no consumo energético de $31,2 \%$ comparado ao sistema convencional, sendo que a energia associada à produção dos materiais utilizados na implantação das melhorias do cenário proposto apresentou pouca contribuição no impacto, levando em conta a vida útil do equipamento.

O consumo de água no processo de destilação foi reduzido de $8 \mathrm{~L}$ no cenário convencional para zero no cenário proposto, representando uma economia de $83,2 \mathrm{~m}^{3}$ de água da concessionária e $854 \mathrm{k}$ Wh de eletricidade da rede de distribuição por ano. A economia tende a aumentar se a produção de água destilada for maior do que aquela adotada no estudo, já que a energia associada à infraestrutura tende a se diluir mais por unidades de produto.

A implantação do sistema de pré-aquecimento da água com aquecimento solar se mostrou mais vantajoso do ponto de vista ambiental, comparando-o ao resfriador geotérmico, representando a maior parte da demanda acumulada de energia primária economizada no cenário proposto.

\section{REFERÊNCIAS}

${ }^{1}$ GUANAIS, Ana Luiza R. et al. Avaliação energética de um sistema integrado de abastecimento de água. Engenharia Sanitária e Ambiental, v. 22, n. 6, p. 1187-1196, 2017.

2 SEO, Emilia S. et al. Avaliação do ciclo de vida: ferramenta gerencial para tomada de decisão. Interfacehs, v. 1, n. 1, 2006.

${ }^{3}$ ABNT, Associação Brasileira de Normas Técnicas (2009) NBR ISO 14040: Gestão ambiental - Avaliação do ciclo de vida - Princípios e estrutura. Rio de Janeiro.

${ }^{4}$ ABNT, Associação Brasileira de Normas Técnicas (2009) NBR ISO 14044: Avaliação do Ciclo de Vida: Requisitos e Orientações. Rio de Janeiro.

${ }^{5}$ DE MATOS, Samuel C. et al. Adaptação de uma unidade de destilação de água para reaproveitamento do fluido refrigerante interno com materiais de baixo custo. XII CONGRESSO NACIONAL DE MEIO AMBIENTE DE POÇOS DE CALDAS, 2015.

${ }^{6}$ SILVA, GISELE S. et al. Implantação de programas de uso racional da água em campi universitários. In: I CONFERÊNCIA LATINO-AMERICANA DE CONSTRUÇÃO 
SUSTENTÁVEL-X ENCONTRO NACIONAL DE TECNOLOGIA DO AMBIENTE CONSTRUÍDO, Anais. São Paulo, Brasil, 2006.

${ }^{7}$ ABNT, Associação Brasileira de Normas Técnicas (2008). NBR 15569: sistema de aquecimento solar de água em circuito elétrico direto: projeto e instalação. Rio de Janeiro.

${ }^{8}$ DUTRA, Adriane et al. Aproveitamento de fontes Alternativas para redução do consumo de energia elétrica e reflexos nos seus custos. Dissertação, UFSM, Santa Maria - RS, 2011. 